\title{
Status of the Development of Flight Power Processing Units for the NASA's Evolutionary Xenon Thruster - Commercial (NEXT-C) Project
}

\author{
Michael V. Aulisio ${ }^{1}$, Luis R. Piñero ${ }^{2}$, Brandon L. White ${ }^{3}$, and Tyler A. Hickman ${ }^{4}$ \\ NASA Glenn Research Center, Cleveland, OH 44135, USA \\ James J. Bontempo ${ }^{5}$ \\ ZIN Technologies Inc., Cleveland, OH 44130, USA \\ Thomas A. Hertel ${ }^{6}$ \\ Aerojet Rocketdyne, Canoga Park, CA 91309, USA \\ and \\ Arthur G. Birchenough ${ }^{7}$ \\ Vantage Partners LLC, Cleveland, $\mathrm{OH} 44135$, USA
}

\begin{abstract}
A pathfinder prototype unit and two flight power processing units (PPUs) are being developed by the Aerojet Rocketdyne Corporation in Redmond, Washington and ZIN Technologies in Cleveland, Ohio, in support of the NEXT-C Project. This project is being led by the NASA Glenn Research Center in Cleveland, Ohio, and will also yield two flight thrusters. This hardware is being considered to be provided as Government Furnished Equipment for the New Frontiers Program, and is applicable to a variety of planetary science missions and astrophysics science missions. The design of the NEXT-C PPU evolves from the hardware fabricated under the NEXT technology development project. The power processing unit operates from two sources: a wide input 80 to $160 \mathrm{~V}$ high-power bus and a nominal $28 \mathrm{~V}$ low-power bus. The unit includes six power supplies. Four power supplies (beam, accelerator, discharge, and neutralizer keeper) are needed for steady state operation, while two cathode heater power supplies (neutralizer and discharge) are utilized during thruster startup. The unit in total delivers up to $7 \mathrm{~kW}$ of regulated power to a single gridded-ion thruster. Significant modifications to the initial design include: high-power adaptive-delay control, upgrade of design to EEE-INST-002 compliance, telemetry accuracy improvements, incorporation of telemetry to detect plume-mode operation, and simplification of the design in select areas to improve manufacturability and commercialization potential. The project is presently in the prototype phase and preparing for qualification level environmental testing.
\end{abstract}

${ }^{1}$ Electrical Engineer, Power Management and Distribution Branch, 21000 Brookpark Rd / 301-5, Non Member

${ }^{2}$ Senior Electrical Engineer, Electric Propulsion Systems Branch, 21000 Brookpark Rd / 301-3, Associate Fellow

${ }^{3}$ Electrical Engineer, Power Management and Distribution Branch, 21000 Brookpark Rd / 301-5, Non Member

${ }^{4}$ Aerospace Engineer, Electric Propulsion Systems Branch, 21000 Brookpark Rd / 301-3, Non Member

${ }^{5}$ Electrical Engineer, ZIN Technologies, 6745 Engle Rd, Non Member

${ }^{6}$ Electrical Engineer, Aerojet Rocketdyne, 8900 De Soto Avenue, Non Member

${ }^{7}$ Senior Electrical Engineer, Vantage Partners, 3000 Aerospace Pkwy, Non Member 


\section{Nomenclature}

$\begin{array}{ll}A C & =\text { Alternating Current } \\ C D R & =\text { Critical Design Review } \\ D C & =\text { Direct Current } \\ D C I U & =\text { Digital Control Interface Unit } \\ F P G A & =\text { Field Programmable Gate Array } \\ G F E & =\text { Government Furnished Equipment } \\ I P S & =\text { Ion Propulsion System } \\ M L C C & =\text { Multilayer Ceramic Capacitor } \\ N E X T & =\text { NASA's Evolutionary Xenon Thruster } \\ N E X T-C & =\text { NASA's Evolutionary Xenon Thruster - Commercial } \\ P C B & =\text { Printed Circuit Board } \\ P P U & =\text { Power Processing Unit } \\ P W M & =\text { Pulse Width Modulation } \\ S M D & =\text { Science Mission Directorate }\end{array}$

\section{Introduction}

$\mathrm{N}$ ASA's Evolutionary Xenon Thruster Ion Propulsion System (IPS) technology has been in development by the Science Mission Directorate (SMD) for use in a variety of robotic science missions. The IPS hardware to be produced as part of this NEXT-C Project consists of two flight thrusters and two flight PPUs. The PPU engineered during the prior NEXT technology development project is utilized as a basis for the NEXT-C PPU design. This heritage PPU is referred to as the NEXT-C PPU Testbed and is shown in Figure 1. The NASA SMD Planetary Science Division is considering providing the NEXT-C flight hardware as Government Furnished Equipment (GFE) for the

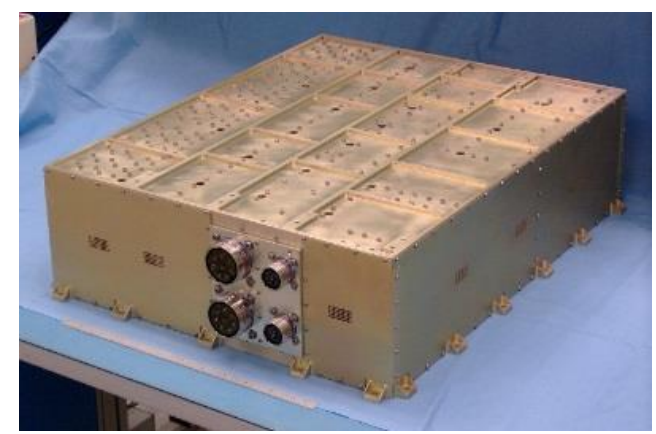

Figure 1. NEXT-C PPU Testbed Hardware
New Frontiers Program. NASA also intends for the NEXT-C system to be available to future users as a commercial product. The outcome of the NEXT-C Project is envisioned to be hardware that can be incorporated as part of both NASA and non-NASA missions.

This paper describes the status of the development of flight power processing units for the NEXT-C Project. An overview of the top-level architecture of the PPU is provided. Multiple improvements made to the heritage design are then described. These improvements include upgrade of design to EEE-INST002 compliance, addition of plume-mode detection circuitry, beam module high-power adaptive-delay control, and improved manufacturability. The present status of the PPU development is then discussed in terms of where it is in the NASA project lifecycle and relative to the availability of two flight PPUs.

\section{Power Processing Unit Overview}

The PPU processes an unregulated or regulated high-power bus input, and provides multiple high-voltage, highcurrent regulated outputs necessary to operate the NEXT-C gridded ion thruster. Figure 2 provides a functional overview of the unit and depicts the electrical interfaces to the thruster. The unit powers a $7 \mathrm{~kW}$ class thruster and is comprised of six power supplies, with the beam (or alternatively referred to as screen) supply being the most prominent as it alone can process in excess of $85 \%$ of the total power. The discharge supply provides power for ionization and processes approximately $10 \%$ of the power. The remaining $5 \%$ is processed by the accelerator, neutralizer keeper, neutralizer heater, and discharge heater supplies; these four power supplies share common primary side switching transistors and are referred to as the quad supply.

The PPU also accepts a low-power bus regulated or unregulated input of 22 to 34 volts. The housekeeping power is derived from this bus. The maximum input power required by the PPU on this bus in approximately 40 watts. An 
RS-485 serial communication interface bus also derives its power from this bus, as this power is included as part of housekeeping.

\section{Power Processing Unit Improvements}

Numerous improvements have been made to the heritage PPU design. The following items will be discussed in the subsequent sections of this paper: the upgrade of components to compliance with EEE-INST-002, incorporation of plume-mode detection circuitry, adaptive-delay control of the beam power supply, and finally improved manufacturability. Improvement in these areas makes the NEXT-C IPS more desirable to prospective missions.

\section{A. EEE-INST-002 Compliance}

The EEE-INST-002 document provides instructions for selection, screening, qualification, and de-rating of EEE parts. ${ }^{1}$ The document defines three levels of parts. The Level 1 parts correspond to the highest reliability and least risk; Level 2 represents low to moderate risk; and Level 3 corresponds to the lowest reliability, highest risk.

Multiple components in the PPU Testbed design were not compliant with EEE-INST-002. Presently, nearly all of the components in the updated NEXT-C PPU design have been selected in accordance with the requirements of EEEINST-002, Level 2. And of these parts, all of them have clear paths to Level 1, enabling a PPU to be fabricated to this most stringent level if a mission so required.

In order to upgrade the design, many non-standard parts were replaced with use as-is EEE-INST-002 parts. Bulk input 500-Volt rated MIL-PRF-49470 MLCC capacitors were incorporated. The capacitors that these MLCCs are replacing from the heritage design made use of a custom dielectric to maximize the stored energy in the available case code size. The custom dielectric exhibited resonances that led to failures of the devices in earlier phases of the technology development project. $^{2}$ The most recently specified standard MLCC capacitors were acquired and screened, and verified to not possess the resonances of their custom dielectric counterparts.

Also related to the upgrade of the design to EEE-INST-002 compliance, limited sections of circuitry were redesigned. The custom housekeeping approach was updated through the use of MIL-PRF-38534 converter modules. Internal board-to-board connectors (inclusive of high-power, low-power, communication, and analog signal) are implemented in the design such that they are compliant with the EEE-INST-002 document.

\section{B. Plume-Mode Detection}

Plume-Mode detection is a new feature in the NEXT-C design. Plume-Mode is an undesirable mode of operation for the thruster, in which propellant is inefficiently used. The NEXT-C PPU contains circuitry that provides an analog telemetry output proportional to the neutralizer keeper AC ripple voltage content. A breadboard of this circuit was created and tested with an engineering model gridded-ion thruster at a NASA GRC vacuum facility as depicted in Figure 3 . The data gathered during this test is still being processed, but preliminary results verify that this circuitry provides telemetry that will enable the spacecraft or digital control interface unit (DCIU) to detect when the thruster is operating in plume-mode.
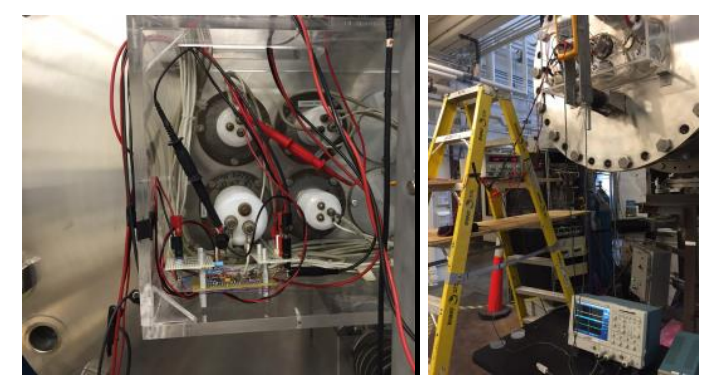

Figure 3. Plume-Mode Testing at NASA GRC 


\section{High-Power Adaptive-Delay Control}

A noteworthy technical challenge of the NEXT-C PPU is the ability of the unit to operate efficiently over a wide range of input and output voltages. This requirement was a significant factor that led to the selection of a phase-shift / pulse-width modulated dual-bridge topology for the beam converter. ${ }^{3,4}$ Control of the mode in which the supply is operating is crucial to the performance of the unit.

The phase-shift mode is required to reach large beam supply output voltages at lower PPU input voltages. In this mode, the beam supply secondary stages effectively operate in series. Conversely, at lower beam output voltages and higher PPU input voltages, the unit operates more efficiently in the traditional PWM mode where the secondary stages operate in parallel. A critical voltage line was defined, such that it

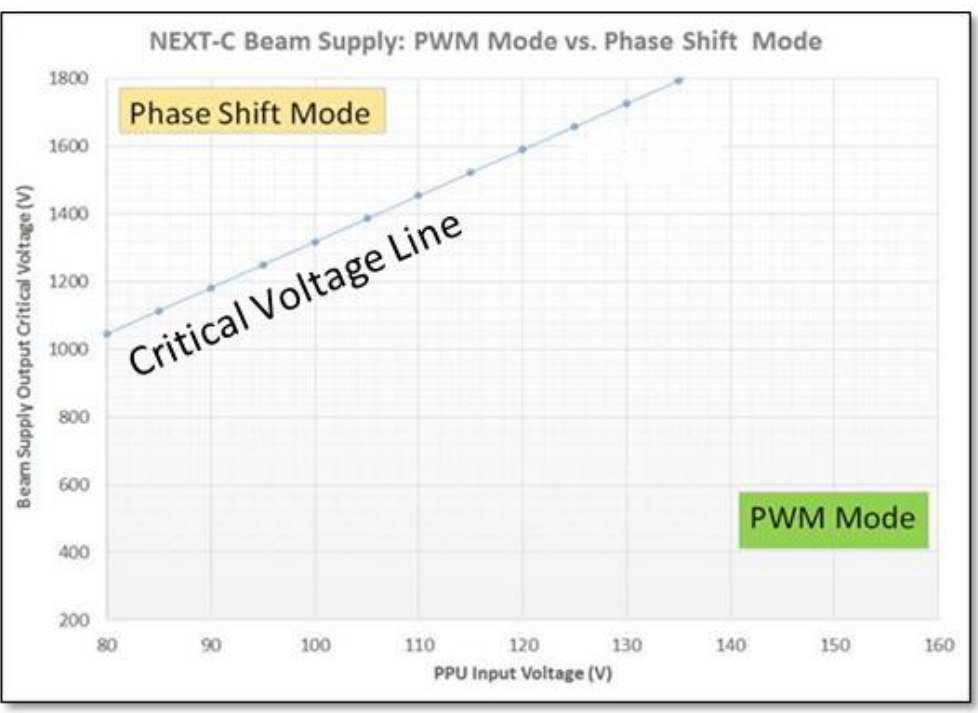

Figure 4. NEXT-C PPU Boundary between Phase-Shift and PulseWidth Modulation. Note that overlap exists between modes.

represented a theoretical boundary (or for practical purposes an area) between PWM and phase-shift modes of operation. Figure 4 depicts this concept.

The high-power adaptive-delay control was implemented to insure stable mode changes. In the heritage design, large amounts of hysteresis were used to ensure transitions between modes, but this had the undesired side effect of compromising the ability to operate at low input voltage. The NEXT-C PPU design uses a novel adaptive-delay circuit to provide smooth mode transitions without compromising performance. The new circuit uses large amounts of hysteresis at areas of operation near the critical voltage boundary, and smaller amounts of hysteresis further away from that area. The adaptive control is necessary because it enables achievement of the high-voltage $1800 \mathrm{~V}$ output at the minimum input of 80 -volts.

\section{Improved Manufacturability}

The NEXT-C PPU is a complex piece of power electronics hardware. An important aspect of this effort is to simplify the design in targeted areas, and thereby improve the manufacturability. The heritage unit was comprised of over 50 discrete printed circuit boards (PCBs) and required more than 130 custom magnetic components. Table 1 highlights the reduction in both PCBs and custom magnetic device count. The decrease in custom magnetic component count is largely due to the replacement of these custom devices with off-the-shelf MILSTD-981S magnetics and MIL-PRF-38534 hybrids where it is logical to do so.

Another area in which the manufacturability has been improved is internal interconnects. Multiple board-to-board interconnections have been eliminated through the use of space-grade flex board technology. This has also enabled reduction in the overall circuit board count, as some power and control boards have been combined into a single flex board. Another example of improved internal interconnects is in the area of the high-current distribution on the highpower bus. In the heritage design, a hand-soldered approach was employed where multiple wires were directly 
soldered to the power distribution bus. The distribution has been improved by utilizing high-current/high-voltage EEE-INST-002 approved sub-miniature-D style connectors.

\section{E. Improved Telemetry, Fault Detection, and Worst-Case Analysis}

The NEXT-C PPU includes over 20 unique voltage, current, and temperature telemetry points that provide essential PPU information to the mission spacecraft. Many of the telemetry circuits were improved, and the ZIN Technologies team performed a worst-case analysis to verify that the telemetry circuits meet the system accuracy requirements. The fault detection systems within the PPU were also improved. The heritage PPU combined all of the possible faults into one signal to the field programmable gate array (FPGA). The NEXT-C PPU incorporates an updated FPGA design that can report several unique faults, greatly improving the ability the system to determine the operating status of the PPU and thruster.

\section{Power Processing Unit Present Status}

The NEXT-C PPU is presently in the prototype phase, preparing for qualification-level environmental testing. The PPU successfully completed Preliminary Design Review in February 2016 and System Requirements Review in July 2015. The Critical Design Review (CDR) is scheduled to occur in March/April of 2017 upon completion of system integrated testing. The flight hardware phase of the project will commence immediately following CDR.

Currently, the NEXT-C Prototype PPU electrical and mechanical designs are complete. The prototype consists of 20 unique printed circuit boards, and seven mechanical subassemblies. The team has fabricated approximately $80 \%$ of the bare printed circuit boards, and approximately $50 \%$ of the mechanical subassemblies. An example of a populated NEXT-C power distribution board is shown in

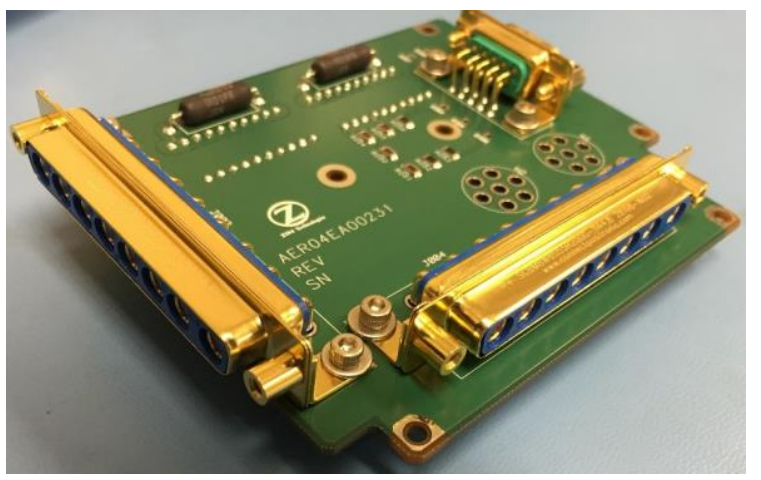

Figure 5. Power Distribution PCB for the NEXT-C Prototype PPU Figure 5.

The NEXT-C Prototype PPU assembly is on schedule to be completed in the third quarter of calendar year 2016; the unit will have completed the environmental tests of EMI, shock, vibration, and thermal vacuum in the fourth quarter of 2016. The Prototype PPU will be integrated with the NEXT-C Thruster in a system-level test at NASA GRC in the first quarter of 2017.

\section{Summary}

The NASA Glenn Research Center is leading the NEXT-C Project to produce two flight power processing units and two flight gridded-ion thrusters. ZIN Technologies and Aerojet Rocketdyne are developing and fabricating the hardware, and many improvements have been made to the heritage PPU that serves as a basis for the design. NASA is considering offering the hardware to the New Frontiers Program. The flight qualified designs that are the outcome of this effort are intended to be used in both NASA and non-NASA missions.

\section{Acknowledgments}

The authors would like to thank the NASA SMD Planetary Science Division for their support in this effort.

\section{References}

${ }^{1}$ EEE-INST-002: Instructions for EEE Parts Selection, Screening, Qualification, and Derating, NASA/TP-2003-212242, May 2003. 
${ }^{2}$ Soeder, James F.; Pinero, Luis; Scheidegger, Robert; Dunning, John; Birchenough, Arthur, "NASA's Evolutionary Xenon Thruster (NEXT) Power Processing Unit (PPU) Capacitor Failure Root Cause Analysis", AIAA 2012-4221, 10 ${ }^{\text {th }}$ International Energy Conversion Engineering Conference, Atlanta, Georgia, July30 - August 1, 2012.

${ }^{3}$ Pinero, L.R., Bond, T., Okada, D., Phelps, K., Pyter, J., Wiseman, S., "Design of a Modular 5-kW Power Processing Unit for the Next-Generation 40-cm Ion Engine," IEPC-01-329, 27 ${ }^{\text {th }}$ International Electric Propulsion Conference, Los Angeles, CA, October 15-19, 2001.

${ }^{4}$ Pinero, L.R., Hopson, M., Todd, P.C., Wong, B., "Performance of the NEXT Engineering Model Power Processing Unit," AIAA-2007-5214, 43 ${ }^{\text {rd }}$ Joint Propulsion Conference, Cincinnati, OH, July 8-11, 2007. 